\title{
PROPRIEDADES DE PROTEÇÃO TÉRMICA DO CARBETO DE SILÍCIO EM REVESTIMENTOS CERÂMICOS
}

\author{
Marcela Galizia Domingues ${ }^{\mathrm{a}, *,(\mathbb{B})}$ e José Atílio Fritz Fidel Rocco ${ }^{\mathrm{a}}$ \\ aDepartamento de Química, Instituto Tecnológico de Aeronáutica, 12228-900 São José dos Campos - SP, Brasil
}

Recebido em 22/06/2020; aceito em 01/09/2020; publicado na web em 30/09/2020

\begin{abstract}
PROPERTIES OF SILICON CARBIDE THERMAL PROTECTION IN CERAMIC COATINGS. In recent years, research on the development of protective materials has expanded significantly in order to find efficient and economically viable solutions for application in various industrial segments. Among these materials, those aimed at protecting metallic substrates against high temperatures that end up causing corrosive processes, stand out due to the huge market demand. Advantages such as reproducibility in the process, cost reduction and reduction in environmental impact also directly contribute to the search for protective coatings as an efficient protective alternative. This article presents the characterization of a ceramic coating developed in a previous study that used silicon carbide as a filler, using potassium silicate (water glass) as a matrix (binder), and whose anti-corrosion and thermal protection properties stood out, mainly in applications in the aerospace industry. In this stage of the work, tests of thermal analysis (thermogravimetry) were carried out for the physical and chemical characterization of the material. The results corroborate those previously achieved when applying the coating on components of a hybrid rocket engine tested on a fixed-point bench.
\end{abstract}

Keywords: thermal protection; silicon carbide; potassium silicate; ceramic material; thermal analysis.

\section{INTRODUÇÃO}

Quando expostos a altas temperaturas, a maioria dos metais sofre um processo de oxidação em uma ampla gama de condições. Questões práticas sobre a vida útil do material e os métodos de proteção contra corrosão centram-se, portanto, na taxa de oxidação e em como controlar a morfologia da reação. Essa última questão demanda uma compreensão mais aprofundada dos processos envolvidos e de como modificá-los. ${ }^{1}$

Quando comparada à corrosão aquosa, as temperaturas consideradas devem ser suficientemente elevadas para que a água, quando presente nos sistemas, esteja no estado de vapor e não como líquido, sendo esta a condição que diferencia o processo corrosivo do oxidativo, que é bastante comum em componentes de turbina a gás, por exemplo. Assim, quando expostos a condições oxidantes (temperaturas entre 100 e $500{ }^{\circ} \mathrm{C}$ ), a maioria dos metais e ligas formam produtos finos de corrosão que crescem lentamente.

Ao projetar materiais metálicos para emprego em temperaturas elevadas, eles devem não apenas resistir o máximo possível aos efeitos produzidos pela reação com o oxigênio, mas também resistir ao ataque de outros oxidantes, já que o ambiente de exposição é, frequentemente, composto por diferentes tipos de materiais em estado gasoso, além de materiais particulados. Diante disso, é mais adequado que se discuta sobre resistência à corrosão a alta temperatura no lugar de resistência a oxidação apenas. ${ }^{2}$

De forma simplificada, processos corrosivos resultam de reações químicas heterogêneas ou eletroquímicas que, normalmente, ocorrem na interface entre o metal e o meio corrosivo, podendo também ser definidos como "todo processo eletroquímico que resulta na degradação e/ou perda de propriedades químicas, físico-químicas ou mecânicas de um material metálico". ${ }^{3}$ Os mecanismos de processos corrosivos dependem do meio corrosivo e do material onde eles se instalam, ocasionando diferentes tipos de corrosão, tais como: eletroquímica; corrosão em água ou em soluções aquosas; corrosão atmosférica; corrosão em sais fundidos; corrosão química; corrosão seca (desencadeada por exposição a temperaturas elevadas, gases,

*e-mail: marcelagalizia@hotmail.com vapores ou por ausência de umidade); corrosão em solventes orgânicos isentos de água e corrosão de materiais não metálicos. ${ }^{4}$

A instalação gradativa do processo corrosivo pode resultar na destruição parcial ou total do metal, culminando em altos custos relacionados à manutenção e/ou à substituição de infraestruturas e equipamentos. A fim de minimizar tais problemas, materiais do tipo coating (revestimento) têm sido amplamente estudados e empregados em diversos segmentos industriais. ${ }^{5}$ São tratamentos que consistem num processo de revestimento da superfície a ser protegida e cujo objetivo é proporcionar uma camada protetiva capaz de aumentar as resistências química e mecânica e a durabilidade do metal. Garantia e facilidade de reprodutibilidade no processo, redução de custos e baixo impacto ambiental têm contribuído para a implementação desta técnica. ${ }^{6}$

As características de proteção dos materiais empregados contra corrosão são determinadas por um complexo mecanismo que inclui a ação e a combinação de diferentes fatores. Ou seja, o comportamento de um sistema protetivo dependerá das propriedades dielétricas do revestimento, da adesão deste ao substrato, da absorção de água e oxigênio; da penetração de íons, de pigmentos e de aditivos inibidores do envelhecimento do revestimento, de buracos e poros no substrato, das características e estado de conservação da superfície do substrato, do pré-tratamento dado a ele, das condições ambientais e de várias reações eletroquímicas complexas que ocorrem na interface metalrevestimento. ${ }^{3}$ Considerando os fatores citados e a demanda de mercado por produtos protetivos contra corrosão e temperaturas elevadas, foi objetivo desta pesquisa o desenvolvimento de um revestimento anticorrosivo e que atuasse como barreira térmica ${ }^{4}$ para atender às necessidades de proteção de equipamentos cujas condições de trabalho submetem-se a temperaturas da ordem de $1800{ }^{\circ} \mathrm{C}$. A formulação à base de silicato de potássio (aglomerante), empregando como carga o carbeto de silício ( $\mathrm{SiC}$ ), conferiu ao revestimento propriedades mecânicas bem definidas e de alta resistência térmica e química, além de atuação satisfatória como barreira protetiva contra oxidação/corrosão, possibilitando, inclusive, a derivação de seu emprego para o setor aeroespacial. O revestimento foi aplicado, com sucesso, na região do convergente-divergente (garganta) da tubeira de um motor foguete do tipo híbrido, em escala laboratorial. ${ }^{7}$ 
As vantagens desse revestimento estão intimamente ligadas à sua capacidade de aderência aos mais diversos substratos metálicos, mesmo sob severas condições, tais como superfícies intensa e constantemente expostas à umidade e névoas marítimas (salt spray), a choques térmicos (brusca variação de temperatura) e à vibração.

Sua base inorgânica (silicatos), sob a forma de solução aquosa para aplicações em ambientes quimicamente agressivos e altas temperaturas é original devido à característica de suportar condições extremas de operação sem que haja degradação completa do material, indicando a ocorrência de um processo ablativo, além da formação de uma película vítrea que penetra a superfície do substrato na qual é aplicado, passando a fazer parte do mesmo através da formação de uma camada monolítica. Adicionalmente, a cura final do revestimento ocorre de forma simples por meio da troca de calor das superfícies recobertas e expostas a altas temperaturas. Destaca-se que, por recomendação do fornecedor da resina de silicato de potássio, a princípio, a cura deveria ser feita sob exposição à temperatura de $600{ }^{\circ} \mathrm{C}$. Porém, durante os ensaios, observou-se que a cura se dá de forma satisfatória mesmo quando realizada em temperatura ambiente $\left(\sim 25^{\circ} \mathrm{C}\right)$. Essas propriedades foram alcançadas a partir de uma formulação onde o carbeto de silício foi empregado como carga em um aglomerante de silicato de potássio, conferindo ao revestimento a característica de material cerâmico.

A motivação central deste trabalho foram os resultados alcançados durante um Projeto de Pesquisa e Desenvolvimento (P\&D), realizado em parceria com a indústria, no qual as pesquisas iniciais buscavam desenvolver um novo material protetivo anticorrosivo capaz de resistir a temperaturas elevadas. A proposta era que o revestimento pudesse ser aplicado às carcaças das turbinas estacionárias (a gás) de grupos geradores de energia elétrica empregadas como sistemas de exaustão de gases, nos quais as temperaturas de trabalho podem chegar a $700{ }^{\circ} \mathrm{C}$. Essa condição possibilitou a derivação da aplicabilidade do material para componentes um motor foguete o híbrido de bancada de testes, conforme apresentado nos estudos de Domingues e Rocco. ${ }^{7}$

Como parte complementar do estudo, foram realizados ensaios de análise térmica aplicando-se a técnica de termogravimétrica para a caracterização quantitativa do revestimento em termos de decomposição térmica, a fim de melhor entender o desempenho protetivo do material componente, em especial, do principal componente da formulação do revestimento, o carbeto de silício. $\mathrm{O}$ presente artigo apresenta os resultados desses ensaios, que confirmaram aqueles alcançados nos ensaios experimentais da pesquisa.

\section{Capacidade de proteção térmica do $\mathrm{SiC}$}

Enquanto material inorgânico, o carbeto de silício possui propriedades como alta estabilidade termoquímica, alta dureza e tenacidade à fratura, baixo coeficiente de expansão térmica, dentre outras. Essas propriedades permitem que seja amplamente utilizado na fabricação de dispositivos refratários, semicondutores, motores de combustão, etc. ${ }^{8}$

Na década de 1990, o SiC foi alvo de diversos estudos relacionados a materiais resistentes a altas temperaturas ${ }^{9-11} \mathrm{em}$ diferentes contextos. Devido à sua excelente resistência à oxidação, compatibilidade química com diversos materiais e inércia química a maioria dos álcalis e ácidos, configura-se enquanto elemento atraente, podendo ser empregado como carga para revestimentos protetivos contra processos oxidativos e corrosivos. Sua aplicação mais comum é em materiais refratários, não apenas devido ao seu alto ponto de fusão $\left(2730^{\circ} \mathrm{C}\right)$, mas também, pela sua elevada estabilidade e resistência térmica $(\sim 800-1000 \mathrm{MPa})$ e elevados valores de tenacidade à fratura $\left(6-10 \mathrm{MPa} \mathrm{m}^{1 / 2}\right) .{ }^{9} \mathrm{~A}$ Figura 1 apresenta uma curva de análise termogravimétrica de carbeto de silício nanoparticulado, na qual observa-se sua estabilidade térmica a temperaturas de $800{ }^{\circ} \mathrm{C} .{ }^{10}$

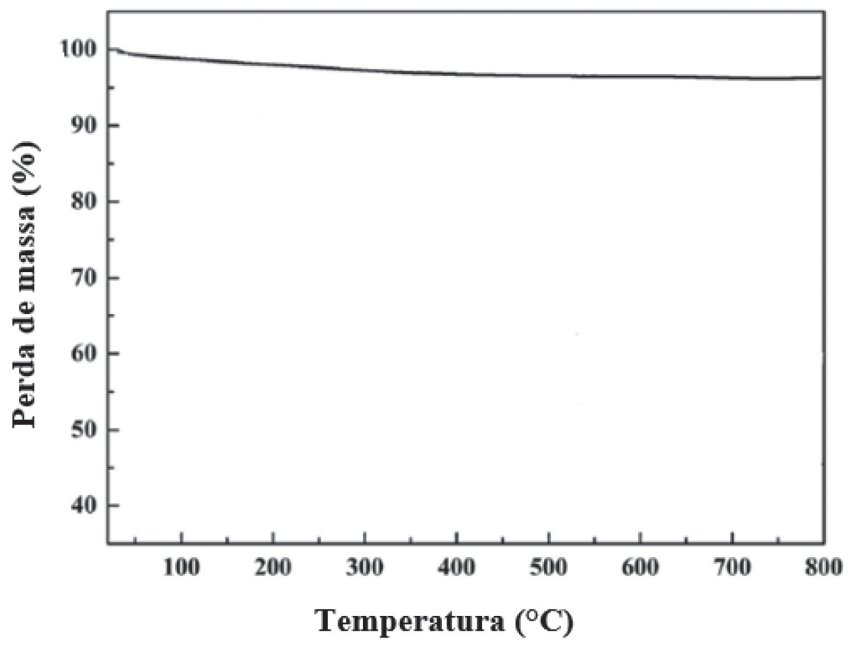

Figura 1. Curva termogravimética de amostra de carbeto de silício nanoparticulado. A razão de aquecimento empregada foi de $10^{\circ} \mathrm{C} \mathrm{m}^{-1}$, a atmosfera empregada foi oxigênio. A vazão de gás e a quantidade de amostra analisada não foram informadas. Adaptado da ref. 10

Em outro estudo realizado por Tishchenko, Ilchenko e Kuzema ${ }^{11}$ sobre a síntese do carbeto de silício, foram realizadas análises térmicas empregando TGA-DTG e TG-DSC, nas quais foi observada uma pequena perda de massa em relação à temperatura. De acordo com os autores, ${ }^{11}$ os resultados das curvas de TGA e DSC de SiC apontam perdas de massa em diversas regiões, sendo a primeira região localizada entre as temperaturas de 30 a $194{ }^{\circ} \mathrm{C}$ (com o pico de perda a $69^{\circ} \mathrm{C}$ ). Essa perda é acompanhada de um evento endotérmico que indica a remoção de água da amostra. Nesta fase, a perda total de massa foi de $0,23 \%$. A perda correspondente à oxidação do carbono foi observada a $325-360{ }^{\circ} \mathrm{C}$. Em seguida, há um aumento da massa da amostra, indicando o início da oxidação do SiC. Entre as temperaturas de 325 a $360{ }^{\circ} \mathrm{C}$, a perda total da amostra foi de $0,04 \%$, o que significa que a quantidade de carbono residual em $\mathrm{SiC}$ não foi menor que esse valor. $\mathrm{O}$ crescimento total da massa durante o aquecimento entre 360 e $1100{ }^{\circ} \mathrm{C}$ foi de $0,23 \%$, o que pode ser uma evidência de que a superfície das partículas de carbeto de silício oxidam-se neste intervalo de temperatura.

O carbeto de silício está sujeito a dois tipos de oxidação: a ativa, que se caracteriza por uma perda de massa contínua e linear, podendo levar o material à completa vaporização, e a passiva, que leva à formação de camada de sílica sobre a superfície do $\mathrm{SiC}$, melhorando o seu desempenho em várias aplicações. ${ }^{11}$

Na maioria dos casos, a oxidação é passiva, ou seja, resulta na formação de uma camada de sílica sobre a superfície do SiC, onde uma ampla variação nas taxas de reação e na morfologia dos produtos da reação pode ser verificada. ${ }^{9}$ Já o processo de oxidação ativa ocorre em pressões de $\mathrm{O}_{2}$ inferiores à 1 bar e pode ser compreendido através da Equação 1:

$$
\mathrm{SiC}(\mathrm{s})+\mathrm{O}_{2}(\mathrm{~g}) \rightarrow \mathrm{SiO}(\mathrm{gO}+\mathrm{CO}(\mathrm{g})
$$

Nessa situação (oxidação ativa), o SiO formado vaporiza após sua formação, levando à perda de massa. Já a oxidação passiva acontece em pressões mais próximas a 1 bar e cuja equação é:

$$
2 \mathrm{SiC}(\mathrm{s})+3 \mathrm{O}_{2}(\mathrm{~g}) \rightarrow 2 \mathrm{SiO}_{2}(\mathrm{~s})+2 \mathrm{CO}(\mathrm{g})
$$


A formação do $\mathrm{SiO}_{2}$ durante a oxidação passiva é depositada sobre a superfície do $\mathrm{SiC}$, levando a um aumento efetivo de massa. Essa deposição atua como um protetor antioxidante e, por consequência, anticorrosivo. $^{12}$

Aliadas às propriedades do carbeto de silício, o emprego de silicato de potássio como aglomerante (binder) conferiu ao revestimento desenvolvido, após processo de secagem (cura), uma alta rigidez resultante da evaporação da água da solução, bem como das diversas reações químicas que ocorrem paralelamente à evaporação de água do meio. Esses processos de estabilização e consolidação são denominados de "silicificação". Numa dessas reações, o silicato (water glass) reage com o dióxido de carbono da atmosfera, conforme apresentado na Equação 3. ${ }^{13}$

$$
\begin{aligned}
& \underbrace{\mathrm{mK}_{2} \mathrm{OnSiO}_{2} \mathrm{xH}_{2} \mathrm{O}}_{\text {water glass }}+\mathrm{mCO}_{2} \underbrace{\mathrm{nSiO}_{2} \mathrm{xH}_{2} \mathrm{O}}_{\text {sílica gel }}+\mathrm{mK}_{2} \mathrm{CO}_{3} \\
& \underbrace{\mathrm{mK}_{2} \mathrm{OnSiO}_{2} \mathrm{xH}_{2} \mathrm{O}}_{\text {water glass }}+\mathrm{Ca}^{++}+\mathrm{mCO}_{2} \rightarrow \underbrace{\mathrm{CaSi}_{\mathrm{n}} \mathrm{O}_{2+1} \mathrm{XH}_{2} \mathrm{O}+\mathrm{mK}_{2} \mathrm{CO}_{3}}_{\text {metassilicato gel }}
\end{aligned}
$$

A sílica gel e o metassilicato gel formados nessas reações complexas contêm uma grande quantidade de água. À medida que essa água evapora, esses géis são convertidos em estruturas sólidas de silicato amorfo, com um baixo teor de água residual, conferindo ao revestimento uma boa resistência química que protege os substratos de intempéries ambientais e temperaturas agressivas, fornecendo, após o processo de cura, um acabamento mate e com baixa rugosidade. ${ }^{13}$

\section{PARTE EXPERIMENTAL}

\section{Caracterização térmica do material}

A análise termogravimétrica vem sendo amplamente utilizada como um método para investigar a decomposição térmica de polímeros e outros materiais, por exemplo, para determinar sua estabilidade térmica. Além disso, uma grande atenção vem sendo dada para a extrapolação dos dados termogravimétricos para determinação de parâmetros cinéticos. ${ }^{14}$

A termogravimetria (TGA) é uma das técnicas de análise térmica que envolve o estudo da variação de massa em função da temperatura e/ou tempo. Com auxílio dessa técnica é possível realizar estudos da cinética de degradação de um material.

Para o estudo do perfil térmico da amostra e determinação dos parâmetros cinéticos da decomposição térmica do revestimento, foram utilizadas as técnicas analíticas de termogravimetria (TGA) e de calorimetria exploratória diferencial (DSC). Os ensaios de TGA foram orientados pela norma ASTM E 1131-08..$^{15}$ Em relação às condições, empregou-se razão de aquecimento de $10{ }^{\circ} \mathrm{C} \mathrm{min}^{-1}$, massa de amostra de $4 \mathrm{mg}$, atmosfera de ar sintético, com vazão de

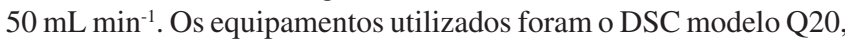
Marca TA Instruments com acessório de resfriamento tipo RCS 40, com porta-amostra de alumínio hermético, e o TGA modelo Q500, Marca TA Instruments, com porta-amostra aberto de platina.

\section{Métodos de determinação dos parâmetros cinéticos}

O estudo da cinética de degradação de um determinado sistema analítico permite encontrar modelos cinéticos que melhor descrevam a reação de degradação e gerar variáveis que auxiliem no estabelecimento do tempo de vida do material em estudo. ${ }^{16} \mathrm{~A}$ degradação de materiais poliméricos decorre de reações químicas ou físicas sofridas pelo material e que podem ser relacionadas ao tipo de aplicação e ao meio ambiente ao qual foi submetido. Esse processo afeta a estrutura do material, alterando suas propriedades. Logo, o conhecimento do estudo cinético a partir da degradação térmica também consiste em um importante fator a ser determinado para diversas aplicações. ${ }^{17,18}$

\section{Método isoconversional}

A cinética não isotérmica pode ser classificada como modelo livre, também chamado de método isoconversional e é mais comumente empregada para realizar a análise cinética de reações no estado sólido. Muitos métodos cinéticos foram propostos para ajustar os dados alcançados com a finalidade de obter os parâmetros que caracterizam o processo de degradação térmica de amostras de espécies químicas. Considera-se a taxa de reação constante na faixa de conversão $(\alpha)$ e dependente somente da temperatura (T). ${ }^{19}$

A cinética das reações químicas em sólidos, mais especificamente a degradação térmica, tal como para a reação genérica, ${ }_{\mathrm{s} 1} \mathrm{~S} 1(\mathrm{~s}) \rightarrow{ }_{\mathrm{s} 2} \mathrm{~S} 2(\mathrm{~s})+\mathrm{gG}(\mathrm{g})$, pode ser descrita pela taxa de conversão do reagente $\mathrm{S} 1$ nos outros, geralmente determinado como uma derivação da lei de ação de massas, originalmente proposto por Guldberg e Waage: ${ }^{20}$

$$
\frac{\mathrm{d} \alpha}{\mathrm{dt}}=\mathrm{k}(\mathrm{T}) f(\alpha)
$$

em que $\alpha$ é o grau de conversão, té o tempo, d $\alpha /$ dt é a taxa de reação, $\mathrm{k}(\mathrm{T})$ é o coeficiente de taxa a uma temperatura absoluta T e $f(\alpha)$ é uma função de $\alpha$, com sua forma exata dependendo da ordem do modelo cinético.

A conversão $\alpha$ da amostra pode ser calculada a partir da perda de massa da amostra durante a reação química (degradação térmica): ${ }^{20}$

$$
\alpha=1-\frac{\mathrm{m}-\mathrm{m}_{\mathrm{f}}}{\mathrm{m}_{0}-\mathrm{m}_{\mathrm{f}}}=\frac{\mathrm{m}_{0}-\mathrm{m}}{\mathrm{m}_{0}-\mathrm{m}_{\mathrm{f}}}
$$

em que $m$ representa a massa da amostra restante no tempo $t ; m_{f}$, a massa final da amostra quando a reação está completa e, $\mathrm{m}_{0}$, a massa inicial da amostra. A constante de velocidade da reação $\mathrm{k}(\mathrm{T})$ pode ser expressa em função da temperatura absoluta $\mathrm{T}$ empregando várias equações. No entanto, a expressão mais usada para $\mathrm{k}(\mathrm{T})$ é descrita na Equação 6:20

$$
\mathrm{k}(\mathrm{T})=\mathrm{A} \exp \left(\frac{-\mathrm{E}_{\mathrm{a}}}{\mathrm{RT}}\right)
$$

em que $\mathrm{A}, \mathrm{E}_{\mathrm{a}}$ e $\mathrm{R}$ representam o fator pré-exponencial, a energia de ativação e a constante universal dos gases, respectivamente. Considerando a relação de Arrhenius para o coeficiente de taxa e temperatura absoluta, a constante de velocidade, Equação 7 pode ser escrita de forma diferencial como:

$$
\frac{\mathrm{d} \alpha}{\mathrm{dt}}=\mathrm{A} \exp \left(\frac{-\mathrm{E}_{\mathrm{a}}}{\mathrm{RT}}\right) f(\alpha)
$$

Para condições não isotérmicas, quando a temperatura absoluta aumenta com o tempo a uma razão de aquecimento constante, $\beta=\mathrm{dT} / \mathrm{dt}$, a Equação 4 pode ser representada como:

$$
\frac{\mathrm{d} \alpha}{\mathrm{dt}}=\frac{\mathrm{A}}{\beta} \exp \left(\frac{-\mathrm{E}_{\mathrm{a}}}{\mathrm{RT}}\right) f(\alpha)
$$

Assumindo que o fator pré-exponencial seja independente da temperatura, pode-se obter a forma integral da equação de grau de conversão: 
$\mathrm{g}(\alpha)=\int_{0}^{\alpha} \frac{\mathrm{d} \alpha}{f(\alpha)}=\frac{\mathrm{A}}{\beta} \int_{T_{0}}^{T} \exp \left(\frac{-\mathrm{E}_{\mathrm{a}}}{\mathrm{RT}}\right) \mathrm{dT} \approx \frac{\mathrm{A}}{\beta} \int_{0}^{T} \exp \left(\frac{-\mathrm{E}_{\mathrm{a}}}{\mathrm{RT}}\right) \mathrm{dT}$

Em que o limite inferior de integração da última integral pode ser aproximado para zero, desde que o grau de conversão seja desprezível para temperaturas abaixo da temperatura ambiente $\mathrm{T}_{0}$.

\section{Método de Flynn-Wall-Ozawa}

A energia de ativação pode ser calculada pelo método isoconversional de Flynn-Wall-Ozawa (FWO) para o estudo da cinética de degradação com o uso de dados de curvas TGA. A vantagem desse método é a determinação da energia de ativação sem que seja necessário conhecer a ordem da reação. Ele assume que a função de grau de conversão $f(\alpha)$ permanece constante para todos os valores de conversão $\alpha$ em diferentes razões de aquecimento $\beta$. A partir do gráfico de $\ln (\beta)$ versus $1000 /$ T obtém-se a energia de ativação Ea. ${ }^{21,22}$

Para FWO, emprega-se a Equação 9 e a aproximação de Doyle, ${ }^{14}$ e o resultado da integração depois de aplicar logaritmos é:

$$
\log \beta=\log \left[\frac{\mathrm{AE}_{\mathrm{a}}}{g(\alpha) \mathrm{R}}\right]-2,315-0,457 \frac{\mathrm{E}_{\mathrm{a}}}{\mathrm{RT}}
$$

O erro aproximado da energia de ativação pode ultrapassar até $10 \%$, dependendo do valor de Ea / RT. ${ }^{14}$

\section{RESULTADOS E DISCUSSÃO}

Inicialmente, o levantamento de uma curva TGA-DTG (Figura 2) de uma amostra do revestimento permitiu uma comparação direta com resultados da literatura ${ }^{10}$ sobre o comportamento de decomposição térmica do $\mathrm{SiC}$ ainda que, para o caso do presente estudo, tenhase empregado o silicato de potássio (water glass) como matriz aglomerante.

A partir da curva TGA da Figura 2, observa-se, primeiramente, a resistência à decomposição térmica da amostra que perdeu, aproximadamente, $6 \%$ de sua massa inicial entre as temperaturas de 27,5 e $900{ }^{\circ} \mathrm{C}$. Da observação da curva DTG, é possível afirmar que nesse intervalo de temperaturas foram anotados dois eventos principais: o primeiro relacionado à saída da água não ligada que ocorreu entre a temperatura inicial da corrida até $100{ }^{\circ} \mathrm{C}$, representando $2 \%$ da massa inicial da amostra. O segundo evento ocorre entre as temperaturas de 475 e $650{ }^{\circ} \mathrm{C}$ e deve-se, provavelmente, a uma perda de massa do aglomerante do revestimento (silicato de potássio), também da ordem de $2 \%$ da massa inicial da amostra. Esses resultados coincidem com o estudo de $\mathrm{Hu}$ et al. ${ }^{10}$ que apresenta a curva de uma análise termogravimétrica de carbeto de silício nanoparticulado, na qual sua estabilidade térmica manteve-se até $800^{\circ} \mathrm{C}$.

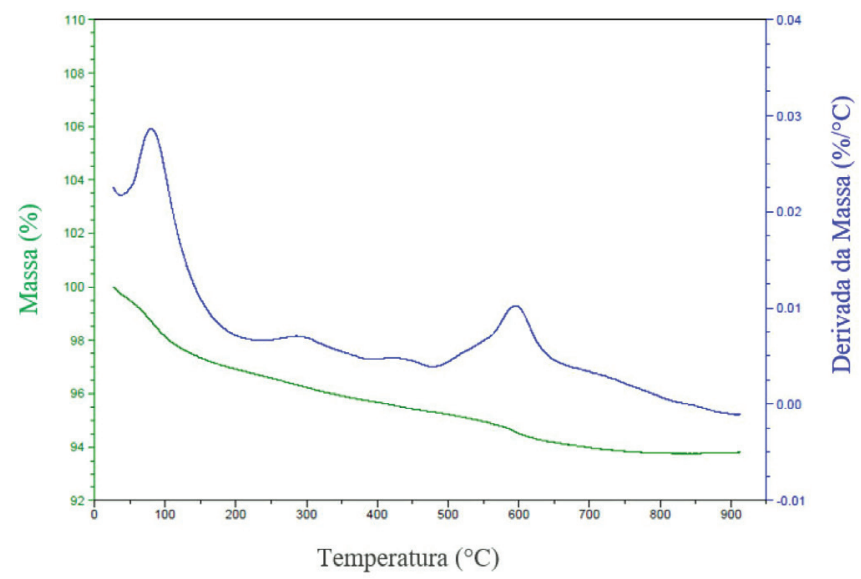

Figura 2. Curva TGA-DTG de uma massa inicial de amostra do revestimento de 3,336 mg para uma razão de aquecimento de $10{ }^{\circ} \mathrm{C} \mathrm{min}^{-1}$ em atmosfera inerte de $\mathrm{N}_{2}$ com vazão de $50 \mathrm{~mL} \mathrm{\textrm {min } ^ { - 1 }}$

Em relação à presença do silicato potássio empregado como aglomerante do revestimento formulado, os estudos realizados por Assaedi, Shaikh, Low, ${ }^{23}$ apontam dois principais eventos de decomposição térmica: um pico em $200{ }^{\circ} \mathrm{C}$ e, outro, em $650{ }^{\circ} \mathrm{C}$. De forma aproximada, a curva TGA apresentada na Figura 2 (acima), guarda similaridade com o evento descrito, uma vez que o segundo pico de perda de massa ocorre entre 475 e $650{ }^{\circ} \mathrm{C}$.

Por se tratar de um revestimento (filme), a coleta das amostras empregadas no levantamento da curva DSC (Figura 4) deveria seguir, a princípio, a recomendação para materiais na forma de filmes (ou membranas), ${ }^{24}$ que estabelece que eles podem ser cortados em pedaços com área suficiente para cobrir a superfície interna do cadinho $\left(\sim 4 \mathrm{~mm}^{2}\right)$, sendo colocados, na sequência, em camadas até atingir a massa adequada para realização do ensaio. No entanto, devido às características do revestimento desenvolvido, em que há uma vitrificação na superfície na qual é aplicado, foi necessário realizar uma raspagem para coletar a amostra. Assim, seguiu-se a recomendação de grânulos, que estabelece que para casos em que a superfície da amostra seja irregular, o grânulo é cortado ou macerado de forma a obter uma superfície plana, desde que não altere o perfil térmico. Após coleta, a amostra é pesada em cadinho de alumínio $(40 \mu \mathrm{L})$ que é, posteriormente, fechado com tampa apropriada.

A título de ilustração sobre a coleta da amostra empregada nos ensaios de termogravimetria, a Figura 3 apresenta o bloco de motor onde foi aplicado o revestimento. A imagem apresenta as etapas de oxidação, preparo de superfície e revestimento aplicado à peça, respectivamente. $\mathrm{O}$ método de preparo de superfície empregado foi o de jateamento (metal quase ao branco), com base na norma NACE $2 .{ }^{25}$ A aplicação do revestimento foi realizada com pincel comum de pintura.
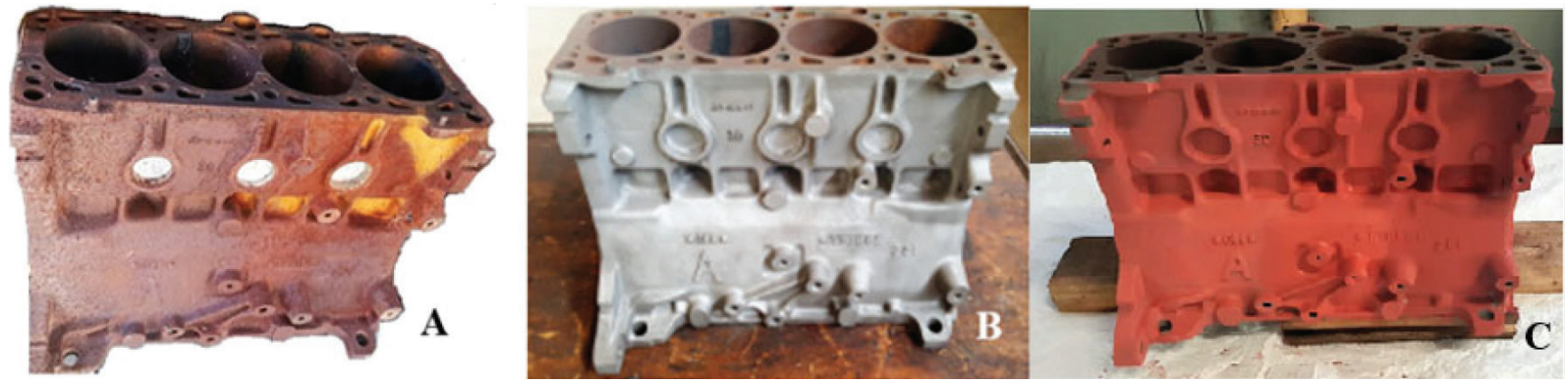

Figura 3. Superfície do bloco do motor de combustão interna oxidada (A), superfície do mesmo bloco do motor preparada para receber o revestimento por jateamento com granalha de aço - grau metal quase ao branco $(B)$ e superfície após aplicação do revestimento protetivo $(C)$ 
A Figura 4 apresenta a curva DSC de massa inicial de amostra do revestimento protetivo de $4 \mathrm{mg}$ para uma razão de aquecimento de $10{ }^{\circ} \mathrm{C}$ por minuto, em atmosfera inerte de $\mathrm{N}_{2}$ com vazão de $50 \mathrm{~mL} \mathrm{m^{-1 }}$. O intervalo de temperatura considerado foi o de 25 a $400{ }^{\circ} \mathrm{C}$ em função da limitação operacional do aparelho DSC empregado nessa análise. As amostras foram colhidas da superfície metálica do bloco motor (Figura 3) que havia recebido o revestimento. A forma de coleta adotada foi a de raspagem, devido às características cerâmicas do material, cuja tendência é vitrificar o filme protetivo. Durante o procedimento de coleta, a amostra pode ter sido contaminada com o material base constituinte do bloco motor (ferro fundido). Isso pode ter interferido nas análises térmicas conforme será apresentado a seguir.

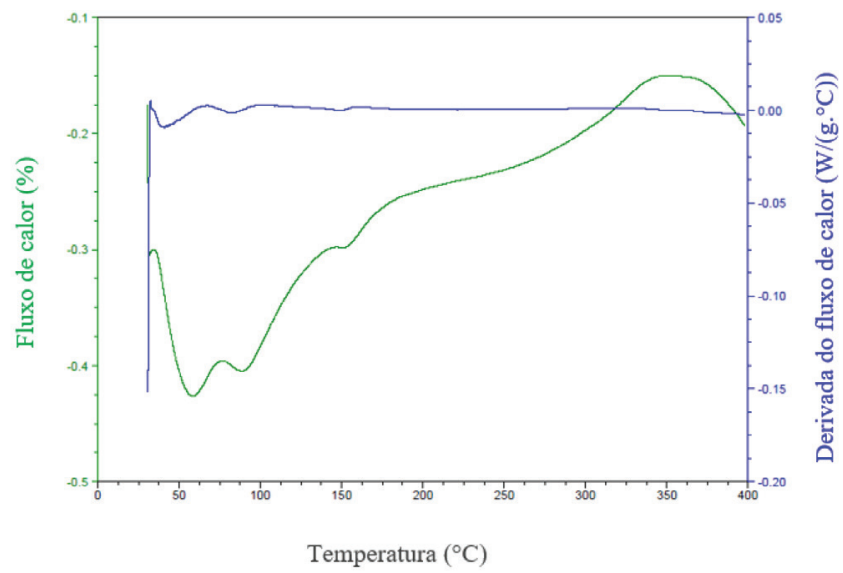

Figura 4. Curva DSC de amostra do revestimento com massa inicial de amostra de 4,1 $\mathrm{mg}$, na razão de aquecimento de $10^{\circ} \mathrm{C} \mathrm{min}^{-1}$, em atmosfera inerte de $\mathrm{N}_{2}$ com vazão de $50 \mathrm{~mL} \mathrm{~min}^{-1}$

A curva da Figura 4 apresenta uma linha base onde observam-se eventos endotérmicos e exotérmicos. A conceituação desses eventos pode ser esclarecida pela curva TGA-DTG da mesma amostra apresentada nessa figura. Visualmente, há uma indicação de que os eventos são de baixa intensidade.

A curva TGA da Figura 5 aponta para eventos distintos que foram devidamente esclarecidos através da identificação das etapas de decomposição térmica da amostra com o emprego da curva de termogravimetria derivada (DTG), em que a derivada da variação de massa em relação ao tempo $(\mathrm{dm} / \mathrm{dt})$ é registrada em função da temperatura ou tempo:

$$
\mathrm{dm} / \mathrm{dt}=f(\mathrm{~T} \text { ou } \mathrm{t})
$$

Portanto, nesse método são obtidas curvas que correspondem à derivada primeira da curva TG, e nos quais os degraus são substituídos por picos que delimitam áreas proporcionais às alterações de massa sofridas pela amostra, facilitando a visualização das etapas das perdas de massa sofridas por meio da precisão de cada um dos eventos.

A primeira etapa da decomposição térmica, que corresponde a uma perda de $4,132 \%$ da massa inicial da amostra, entre as temperaturas de 30,67 e $306,80^{\circ} \mathrm{C}$, refere-se à dois eventos consecutivos: saída da água não ligada do revestimento (até $100{ }^{\circ} \mathrm{C}$ ) e parte da água ligada do silicato de potássio que passou ao estado sólido durante o processo de cura do revestimento (de 100 à $306,8^{\circ} \mathrm{C}$ ). A decomposição térmica dos compostos iônicos depende da propriedade de polarização dos ânions. A polarização é a deformação de ligações iônicas. Ou seja, quanto mais polarizável é o ânion, mais polarizada será a substância e menos estável termicamente será a sua estrutura reticular. A temperatura de decomposição térmica é a energia que

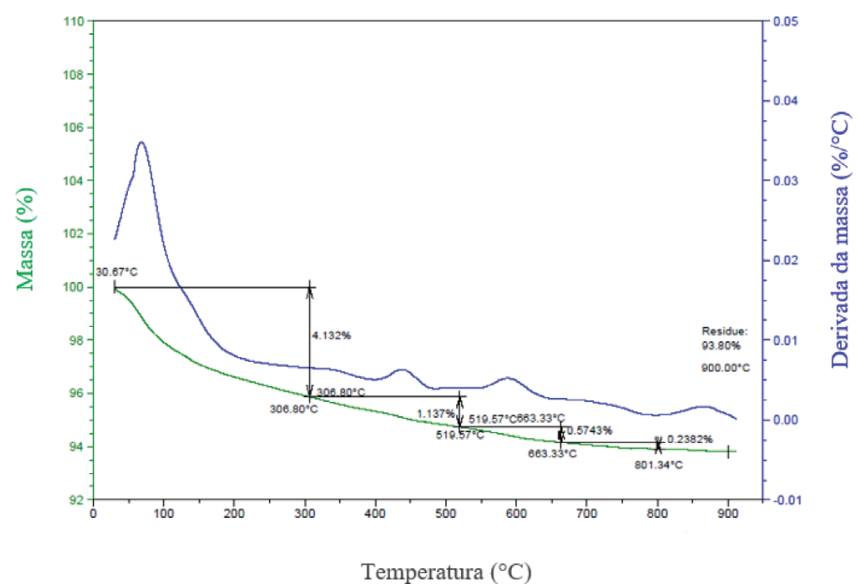

Figura 5. Curva TG/DTG de amostra do revestimento com massa inicial de amostra $4,1 \mathrm{mg}$, na razão de aquecimento de $10^{\circ} \mathrm{C} \mathrm{min}^{-1}$, em atmosfera inerte de $\mathrm{N}_{2}$ com vazão de $50 \mathrm{~mL} \mathrm{~min}^{-1}$

rompe as interações intermoleculares presentes na parte negativa do composto iônico até a decompor em substâncias voláteis menores.

Morsha et al. ${ }^{26}$ apontam a presença de dois tipos de água contida nos cristais: "água ligada" e "água não ligada". A primeira compreende a água na forma de hidroxilas dos hidratos e dos ácidos oxiácidos, e a água molecular dos sais hidratado, enquanto a segunda embora também corresponda à água molecular, não apresenta uma relação definida entre a massa de água e a massa da substância que a contém.

A segunda etapa de perda inicial da amostra do revestimento, entre as temperaturas de 306,8 e $519,57{ }^{\circ} \mathrm{C}$, corresponde à água adsorvida do aditivo anticraquelante representado pela sílica coloidal. Com base na literatura, ${ }^{27}$ esse tipo de sílica possui duas importantes etapas de perda de massa na sua decomposição térmica: a primeira, entre $27{ }^{\circ} \mathrm{C}$ e, aproximadamente, $200{ }^{\circ} \mathrm{C}$ (entre 300 e $\sim 43 \mathrm{~K}$ ), correspondendo às moléculas de água fisicamente adsorvidas na superfície; a outra, entre 325 e $896{ }^{\circ} \mathrm{C}$ (entre 600 e $~ 1170 \mathrm{~K}$ ), que corresponde à condensação dos grupos silanóis. De nome comercial Bindzil ${ }^{\circledR}$, essa sílica participa na formulação do revestimento com aproximadamente $1 \%$ massa/massa.

Infere-se que as duas etapas subsequentes de perda de massa inicial da amostra, tendo início na temperatura de 519,57 até $801,34{ }^{\circ} \mathrm{C}$, correspondem aos resíduos da superfície metálica (ferro fundido) de onde a amostra do revestimento foi coletada. Conforme apresentado anteriormente, o revestimento estava aplicado na superfície de uma carcaça de motor de combustão interna. De acordo com a literatura, ${ }^{28}$ a pirita (predominante no ferro fundido) apresenta uma curva de decomposição térmica em atmosfera inerte com um evento de perda de massa predominante, cujo início se dá na temperatura de, aproximadamente, $366^{\circ} \mathrm{C}(650 \mathrm{~K})$ e término em, aproximadamente, $576{ }^{\circ} \mathrm{C}(850 \mathrm{~K})$. Embora nesse estudo os ensaios de termogravimetria também tenham sido realizados em atmosfera inerte, há uma possibilidade de que o ferro fundido tenha reagido com o oxigênio presente nos demais compostos presentes na amostra.

Em relação ao parâmetro cinético pelo método de Flynn-WallOzawa, baseado na curva TGA da Figura 5, para o intervalo de temperatura entre 25 e $900{ }^{\circ} \mathrm{C}$, o valor da energia de ativação (Ea) foi de $125 \mathrm{~kJ} \mathrm{~mol}^{-1}$. Esse valor corresponde aos eventos citados anteriormente, que englobam a saída da água ligada e da sílica coloidal.

Skorina e Tikhomirova ${ }^{29}$ analisaram curvas TGA e DTG de silicatos de potássio e sódio, em que observaram etapas de decomposição térmica similares às apresentadas neste trabalho, confirmando que na primeira etapa a água ligada deixa a amostra, 
Temperatura $\left({ }^{\circ} \mathrm{C}\right)$

(a)

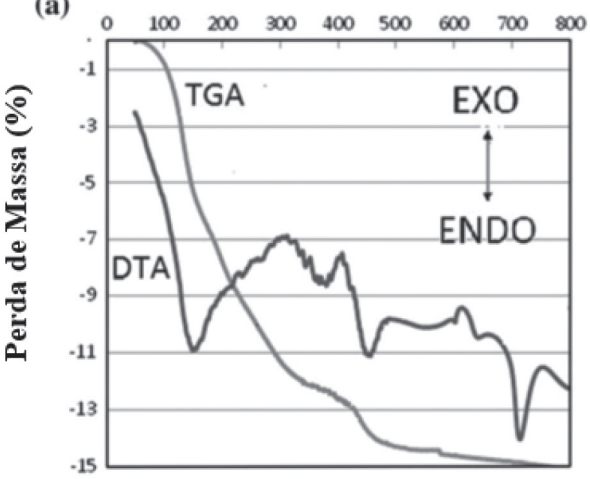

Temperatura $\left({ }^{\circ} \mathrm{C}\right)$

(b)

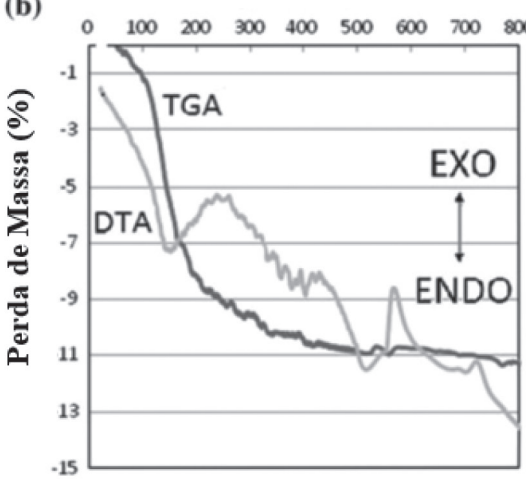

Figura 6. Curvas DTA - TGA comparativas do silicato de sódio usado como ligante (a) e do silicato de potássio usado como ligante (b)

enquanto na segunda etapa quem deixa a amostra é a sílica gel (ela se aproxima do comportamento da sílica coloidal - Bindzil - presente na formulação do revestimento estudado).

Nesse mesmo estudo, ${ }^{29}$ as autoras compararam as curvas DTA do ligante de silicato de sódio e do ligante de silicato de potássio, conforme apresentado na Figura 6.

Observa-se que no silicato de potássio, a perda de massa que acompanha a remoção de água adsorvida na temperatura de 25 a $140{ }^{\circ} \mathrm{C}$, é de 4,6\%, muito próximo à perda de massa do silicato de sódio nessa mesma faixa de temperaturas. No entanto, dentro da faixa de 140 a $560{ }^{\circ} \mathrm{C}$, a perda de massa da amostra de silicato de potássio (Figura 6b) é de $6 \%$, em contraste com uma perda de $10 \%$ observada na amostra silicato de sódio (Figura 6a). De acordo com a literatura, ${ }^{30}$ a eliminação de grupos de hidroxilas associados à superfície de sílica coloidal ocorre na faixa de temperatura de, aproximadamente, 150 a $800^{\circ} \mathrm{C}$. Uma vez que grupos de hidroxilas são encontrados na superfície de partículas de gel (sílica coloidal), a diminuição da área superficial específica do gel resulta na diminuição do número de grupos de hidroxilas.

Os resultados da literatura, ${ }^{29} \mathrm{em}$ que as microestruturas, a composição de fases e a estabilidade hidrolítica do silicato de sódio e do de potássio foram comparados, indicaram o silicato de potássio como material mais adequado para uso como aglomerante (matriz) em situações onde são demandadas resistências química e mecânica. Esses resultados corroboram com aqueles alcançados no presente estudo, tanto nos ensaios experimentais, como aqueles obtidos nas análises térmicas, através da técnica de termogravimetria.

\section{CONCLUSÕES}

O comportamento da decomposição térmica de amostras do revestimento formulado neste trabalho confirmou os resultados experimentais obtidos em testes de bancada, tanto nas aplicações em componentes de um motor foguete híbrido, como na carcaça de um bloco de motor de combustão interna. A perda de massa observada nas curvas termogravimétricas de amostras da formulação citada demostraram sua alta capacidade de suportar condições extremas de calor com mínima degradação do filme aplicado nas superfícies protegidas. De forma geral e com base na literatura comparada, essa perda de massa representou a saída da água ligada e, eventualmente, de parte da água não ligada. O revestimento permaneceu estável até $400{ }^{\circ} \mathrm{C}$, tendo sido observada, até essa temperatura, apenas a saída de umidade e de voláteis.

\section{REFERÊNCIAS}

1. De Souza, R. B.; Reboita, M. S.; Werle, A. P.; Costa, E. B. C.; Revista Eletrônica de Engenharia Civil 2016, 13, 1.
2. Belem, M. J. X.; Fals, H. C.; Lima, C. R. C.; Soldag. Insp. 2015, 20, 661.

3. de Oliveira, A. R.; Corrosão e tratamento de superfície, UFMS: Santa Maria, 2012.

4. Frauches-Santos, C.; Albuquerque, M. A.; Oliveira, M. C. C.; Echevarria, A.; Rev. Virtual Quim. 2013, 6, 169.

5. Zhang, F.; Ju, P.; Pan, M.; Zhang, D.; Huang, Y.; Li, G.; Li, X.; Corros. Sci. 2018, 144.

6. Ulaetoa, S. B.; Rajana, R.; Pancreciousa, J. K.; Rajana, T. P. D.; Paia, B. C.; Prog. Org. Coat. 2017, 111, 294.

7. Domingues, M. G.; Rocco, J. A. F. F.; Int. J. Energ. Mater. Chem. Propul. 2018, 16, 165.

8. Cheng, G.; Chang, T.; Qin, Q.; Huang, H.; Zhu Y.; Nano Lett. 2014, 14, 754.

9. Roy J.; Chandra S.; Das S.; Maitra S.; Rev. Adv. Mater. Sci. 2014, $38,29$.

10. Hu C.; Li Y.; Zhang, N.; Ding, Y.; R. Soc. Chem. Adv. 2017, 7, 11732.

11. Tishchenko, I. Y.; Ilchenko O. O.; Kuzema, P. O.; Chem., Phys. Technol. Surf. 2015, 6, 216.

12. Li, W.; Zhu, S.; Chen, M.; Wang, C.; Wang, F.; Appl. Surf. Sci. 2014, 292, 538.

13. Story, D. Em Paints, Coatings and Solvents, $2^{\text {nd }}$ ed. (completamente revista), Story D.; Werner. F., eds.; Wiley-VCH: Weinheim, 2008, cap. 2.

14. Flynn, J. H.; Wall, L. A.; Polym. Lett. 1996, 4, 487.

15. ASTM - American Society for Testing Materials. ASTM E1131 - 20. Standard Test Method for Compositional Analysis by Thermogravimetry. West Conshohocken, PA, 2020.

16. Bandeira, C. F.; Pereira, A. C.; Botelho, E. C.; Costa, M. L.; J. Mater. Res. 2013, 28, 3094.

17. Santos, D. C.; Jesus, L.F.S.; Santana, P. L.; Calado, M.V.A.; Biscaia Jr, E. C.; Pagano, R.L.; Sci. Plena 2016, 12, 1.

18. Rosa, E. C. A.; Gonçalves, R. F. B.; Domingues, M. G.; Almeida, L. E. N.; Silva, A. C.; Rocco, J. A. F. F.; Quim. Nova 2019, 42, 1678.

19. Fernandez, A.; Mazza, G.; Rodriguez, R.; J. Environ. Chem. Eng. 2018, 6,404 .

20. Soria-Verdugo, A.; Goos, E.; García-Hernando, N.; Riedel, U.; Algal Res. 2018, 32, 11.

21. Parcheta, P.; Koltsov, I.; Polym. Degrad. Stab. 2018, 151, 90.

22. Wang, H.; Yang, J.; Long, S.; Wang, X.; Yang, Z.; Li, G.; Polym. Degrad. Stab. 2004, 83, 229.

23. Assaedi. H.; Shaikh F. U. A.; Low I. M.; Materials (Basel) 2019, 12, 3485.

24. ASTM - American Society for Testing Materials. ASTM D3418 - 15. Standard Test Method for Transition Temperatures and Enthalpies of Fusion and Crystallization of Polymers by Differential Scanning Calorimetry. West Conshohocken, PA, 2015. 
25. NACE Standards. NACE No. 2/SSPC-SP 10-2006-SG, Near-White Metal Blast Cleaning. Product Number: 21066-SG. ISBN: 1-57590108-0.

26. Morscha, S.; Lyona, S.; Greensmithb, P.; Smithc, S. D.; Gibbond, S. R.; Prog. Org. Coat. 2015, 75, 293.

27. Rebelo, M. M.; Nascimento, L. D.; Corrêa, J. A. M.; Cerâmica 2015, 61,359 .
28. Yani S.; Zhang D.; Fuel 2010, 89, 1700.

29. Skorina, T.; Tikhomirova, I.; J Mater Sci. 2012, 47, 5050.

30. Gomes, L. S.; Furtado, A. C. R.; Souza, M. C.; Rev. Virtual Quim. 2018 10,1018 . 